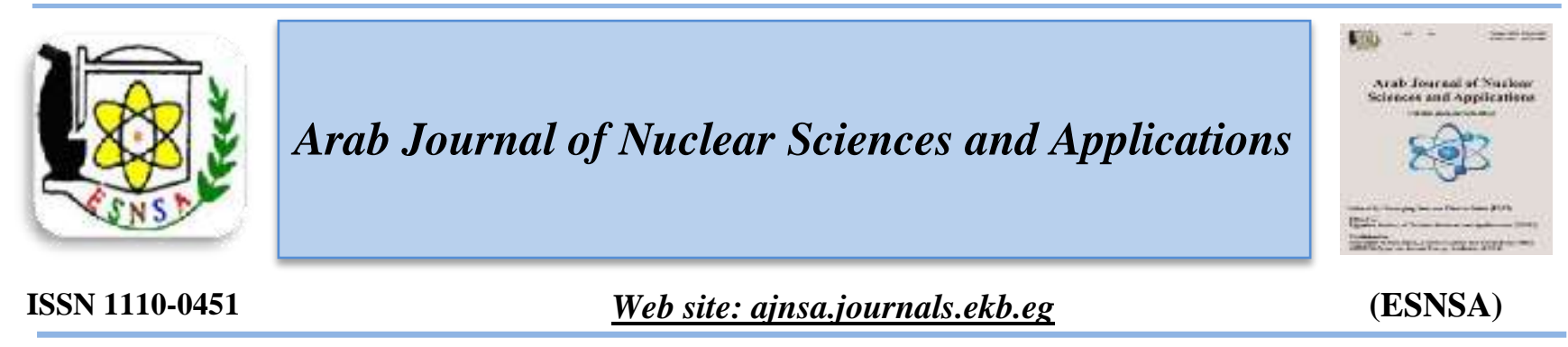

\title{
Spatial Cone Beam Computed Tomography (CBCT) Radiological Findings of Florid Cemento- Osseous Dysplasia: Case Report and Review of Literature
}

\author{
Faten A Alluqmani ${ }^{1 *}$, Hadeel M Alameer ${ }^{1 *}$, Khairiah A Madani $^{1 *}, \operatorname{Radwa}_{\text {A Sallam }}{ }^{2}$, Eman A \\ Arnout $^{3}$ \\ ${ }^{(1)}$ BDS, college of dentistry, Taibah University, Al-Madinah Al-Munawarrah, KSA \\ ${ }^{(2)}$ Health Radiation Research Department, National Center for Radiation Research and \\ Technology (NCRRT), Atomic Energy Authority, Cairo, Egypt \\ (3) Oral Medicine, Periodontology, Diagnosis and Oral Radiology, Faculty of Dentistry, Ain- Shams \\ University, Cairo, Egypt, \\ Departments of Oral and Basic Clinical Sciences, College of Dentistry, Taibah University, Al-Madinah \\ Al-Munawarrah, KSA
}

Received 11 March 2020 Accepted 24 Aug. 2020

Florid cemento-osseous dysplasia (FCOD) is a condition that characteristically affects the jaws of middle-aged black women. Cemento-osseous dysplasia also has been classified into periapical, focal, florid and familial cemento-osseous dysplasia. Radiographically, FCOD appears as a well-defined radiopaque, symmetrical mass lobules, located in the apical part of the teeth with encapsulated margin. Cone-Beam Computed Tomography (CBCT) is a clinical application of ionizing radiation. CBCT is widely used in dental and maxillofacial imaging. This technology has been used for diagnosis and treatment planning for both adult and pediatric patients in orthodontics. It is advantageous being reconstructed in three dimensions, image quality, a 1:1 ratio that allowed reliable dimensions, the possibility for craniofacial imagining, and lower radiation doses compared to traditional CT.CBCT is beneficial in diagnosis of this lesion with perfect evaluation of the effect on the surrounding structures. This paper presents two cases of black female patients who were radiographically diagnosed with FCOD

Keywords: cemento-osseous dysplasia, cone-beam computed tomography, focal cemento-osseous dysplasia, periapical cemento-osseous dysplasia, florid cemento-osseous dysplasia

\section{Introduction}

Cone-Beam Computed Tomography (CBCT) is a clinical application of ionizing radiation. CBCT is advantageous for being reconstructed in three dimensions, image quality, a 1:1 ratio that allowed reliable dimensions, the possibility for craniofacial imagining, and lower radiation doses compared to traditional CT $[1,2]$

Throughout the previous years, CBCT is widely used in dental and maxillofacial imaging. This technology has been used for diagnosis and treatment planning for both adult and pediatric patients in orthodontics. [3]

Fibro-Osseous lesions [FOL] are a group of lesions which are known to affect the jaws and the craniofacial bones which is regarded as a very confusing area in diagnostic pathology . These Fibro-Osseous lesions, according to pathogenetic mechanisms, are classified into Bone dysplasiasa, Cemento-osseous dysplasiasa, Inflammatory/reactive Metabolic Disease and Neoplastic lesions [4]

Corresponding author: radwasallam36@gmail.com

DOI: 10.21608/ajnsa.2020.25704.1336

CScientific Information, Documentation and Publishing Office (SIDPO)-EAEA 
Florid osseous dysplasia is a rare type of Cementoosseous dysplasiasa. The term florid is used because of its widespread and extensive manifestation [5-7]. Cemento Osseous dysplasia are non-neoplastic idiopathic processes, originating from periodontal ligament located in the toothbearing jaw areas, characterized by replacement of normal bone with metaplastic bone and fibrous tissue [7] .

In 1992, the World Health Organization classified cementomatous lesions ${ }^{[6]}$ on the basis of sex, age, radiographic, histopathologic and clinical characteristics. This is in addition to the location of the lesion [6] and familial origin which occurred in some cases $[8,9]$. On the other hand, the process may be totally asymptomatic and accidentally discovered by routine radiographs [10].

Other conditions may show drainage accompanied by exposure of sclerotic calcified masses in the oral cavity or dull pain. This occurs due to progressive alveolar atrophy under a denture or after extraction of teeth in the affected area [11].

Radiographically, the lesions appear as a multifocal well-defined radiopaque mass with encapsulated sclerotic borders located in two or more quadrants, usually in the tooth-bearing areas. They are regularly restricted within the alveolar bone [12].

The present study describes two cases of patients who were clinically and radiographically diagnosed of florid cemento-osseous dysplasia.

\section{Ethical consideration}

This study is considered an Observational Descriptive Study (case report). Verbal and written informed consents are obtained from cases involved in the study. Confidentiality of data was confirmed by the obligation of the principal investigator, and by coding all study subjects included in this study.

\section{Case report 1}

A 30 years-old Female was presented for orthodontic treatment. She was a systemically free and underwent normal extra-oral examination. Intraoral examination revealed multiple carious teeth and the overlying gingiva was normal without any clinical signs of inflammation. The radiographic picture is shown as described in Figures 1, 2, $3 \& 4$.

\section{Case report 2}

A 23 years-old female was presented for orthodontic treatment. She was a systemically free and normal extra-oral examination. Intraoral examination revealed multiple carious teeth and broken teeth. The Routine panoramic radiograph showed well-defined multiple radio-opaque masses on the mandibular left region: periapical to 2 nd molar and interdental between premolars with encapsulated border suspecting focal cementoosseous dysplasia. Although the trabeculae on the mandibular right molar region have been lost, it is of a normal ladder pattern. However, it is still needed to be followed up. Figure 5 .

\section{Discussion}

Cemento-osseous dysplasia are classified based on their radiological intrabony extension into three main groups; periapical, focal (single lesion) and florid (sclerotic symmetrical masses) cemental dysplasia. This disorder is "strictly localized to tooth-bearing areas and not associated with any other skeletal disease" [13].

The pathogenesis of the condition is not clear, however, it has been suggested that it is a dysplastic change or reactive in the periodontal ligament. The diagnosis of FCOD is based mainly on radiographic and clinical findings. FCOD is characterized by the replacement of bone by varying degrees of mineralization connective tissue matrix in the form of woven bone or cementumlike round basophilic acellular structures [14]. The affected area changes from vascular bone into a cementum-like lesion.

The key radiographic features of FCOD are multiple well-defined mixed radiolucent/ radiopaque or dense, cementum-like radiopacities mass that is encapsulated. This encapsulation is characterized by the presence of radiolucent $\mathrm{rim} / \mathrm{band}$ and sclerotic borders [15].

From the Clinical aspect, localized enlargement of the cortical plates might occur, however, FCOD are asymptomatic. Consequently, it requires no treatment except cases in which the lesion causes cosmetically concerning or in cases of secondary infections .Treatment of secondary infection of this lesion can be complicated and difficult $[13,14]$.

In the present two cases, patients were seeking orthodontic treatment. .FOCD was accidently discovered. Diagnosis was based mainly on radiographic findings since the two cases were asymptomatic. No biopsy was taken to avoid precipitation of infection that is difficult to control without extensive surgical intervention 
Based on the cases diagnosis of FOCD, Orthodontic treatment of these cases was contraindicated. This was attributed to the

Moreover, to avoid contamination of the affected bone that could occur during orthodontic treatment, patients and their families should be difficulty in tooth movement in the affected areas because of the disorganization of the alveolar bone process.

aware of the condition to maintain unnecessary treatments that could result in infection of affected areas [16].

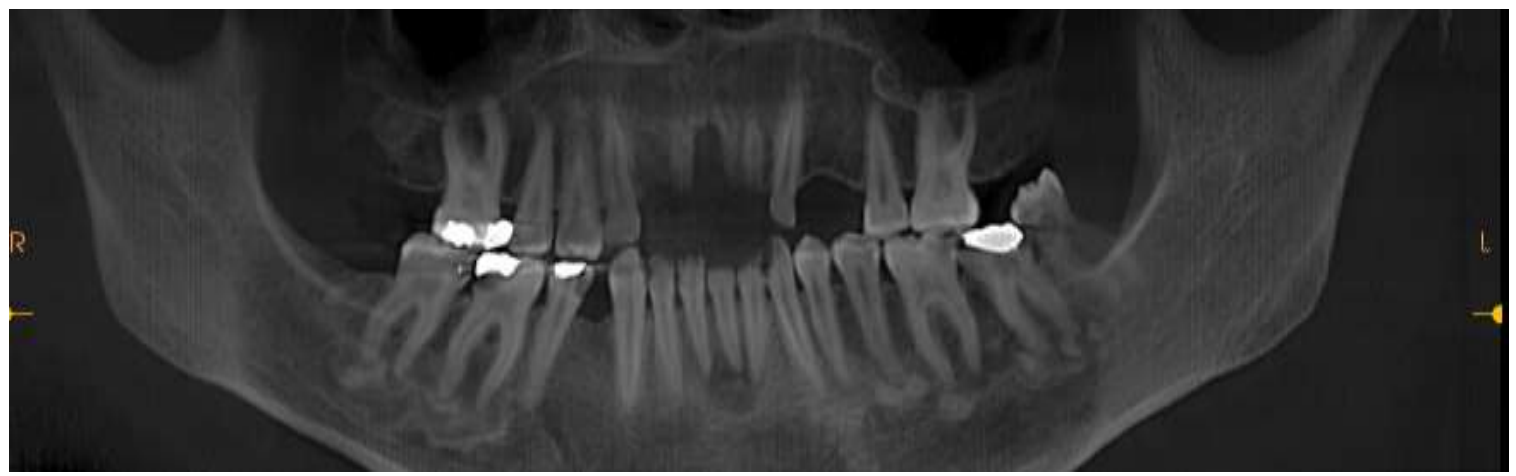

Fig. (1): CBCT reformatted panoramic image showing multiple well-defined radiopaque masses with radiolucent boundaries and has apparent sclerotic border. The lesions bilaterally related to the apices of premolar/ molar region in the mandible and one lesion at the 2nd maxillary right molar area.

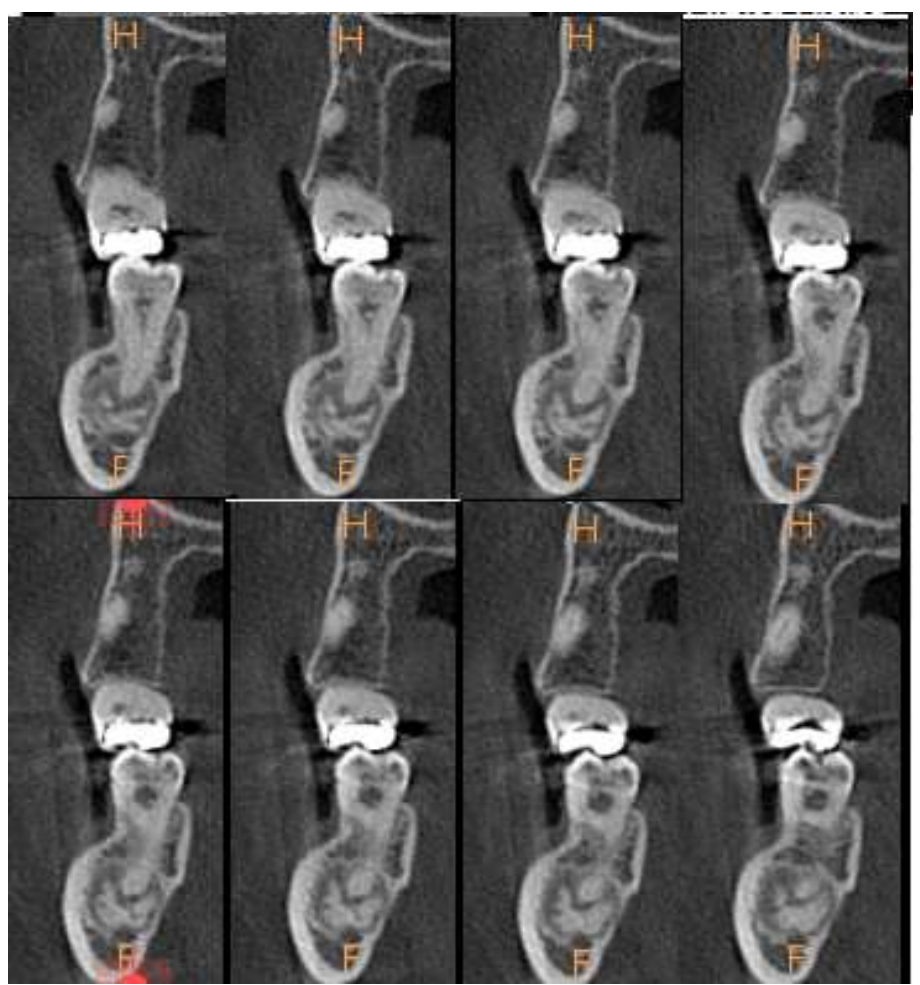

Fig. (2): A successive CBCT sagittal slices among right 2nd molar in maxilla and mandible showed periapical well-defined multiple lobulated radiopaque foci with encapsulated margin. A slight thinning of buccal cortical plate of bone in maxilla and mandible and thinning of lingual cortical bone of mandible 


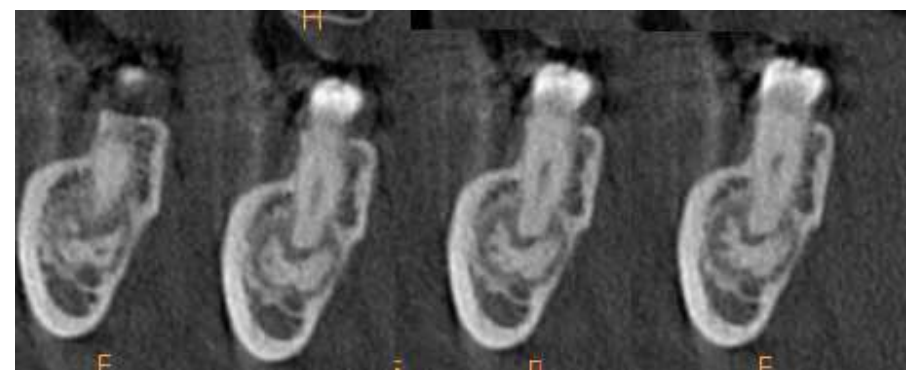

Fig. (3): A successive CBCT sagittal slices along with mandibular 2nd right premolar showed slight displacement of inferior alveolar canal inferiorly.

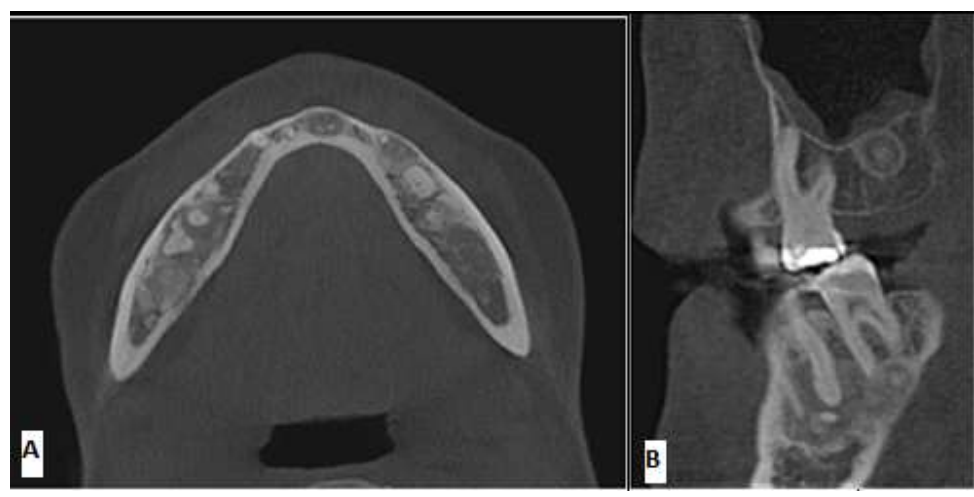

Fig. (4): A, is an axial CBCT slice at level apically to the root apex which show the sclerotic borders around the lesion. B, Sagittal CBCT slice showed the maxillary lesion and sclerotic border of the lesion on the mandibular right side.

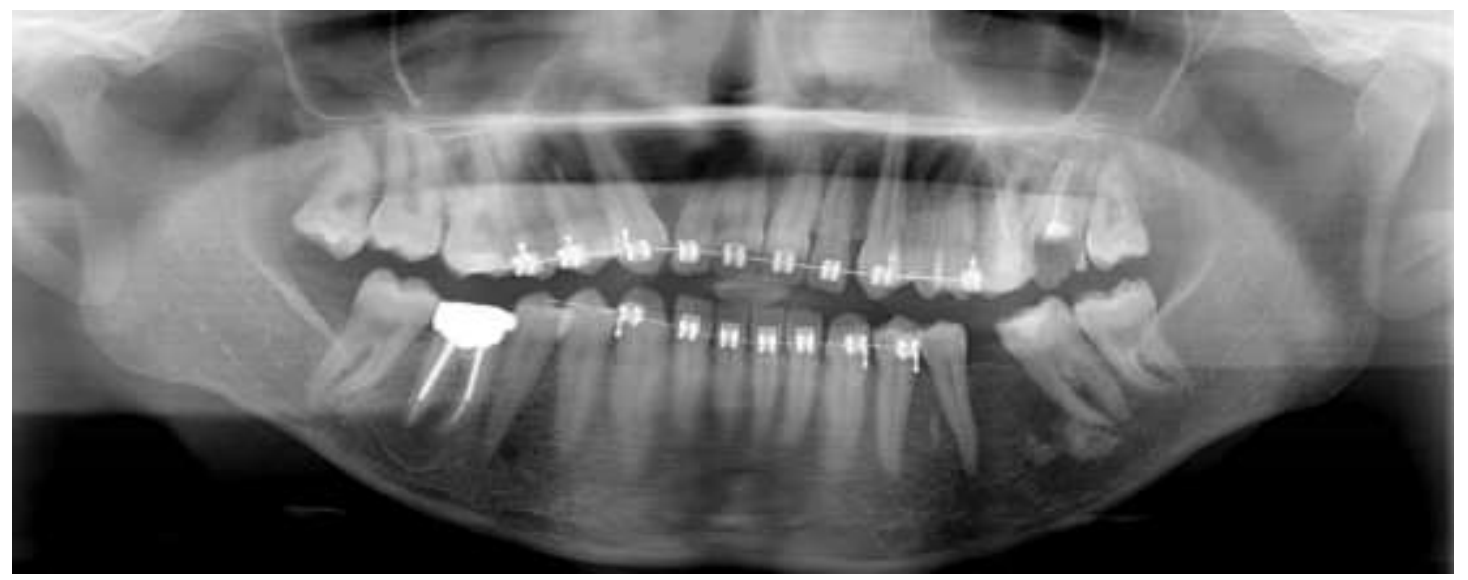

Fig. (5): Panoramic radiograph showed well-defined periapical multiple radio- opaque masses related to mandibular left second molar with encapsulated border. In addition, there is small radio-opaque foci located inter- dental between mandibular left premolars.

\section{Conclusion and Recommendations}

In spite of the biological hazards of $\mathrm{x}$-ray, it is still of a great importance in detecting the silent intrabony lesions such as the present cases of FCOD. It is very essential to make CBCT for detecting FCOD in order to check the exact extension of the lesion and to evaluate the effect of the lesion on the adjacent structures. An annual radiographic follow up is important to assure the persistent benign behavior of the condition.

\section{Conflict of interest}

All authors declare that they have no financial or non-financial conflict of interest in the subject matter or materials discussed in this manuscript. 


\section{Authors' contribution}

All authors contributed equally to the present study.

\section{Reference}

1. Scarfe, W. C., Farman, A. G., \& Sukovic, P. (2006). Clinical applications of conebeam computed tomography in dental practice. Journal-Canadian Dental Association, 72(1), 75.

2. Lorenzoni, D. C., Bolognese, A. M., Garib, D. G., Guedes, F. R., \& Sant'Anna, E. F. (2012). Cone-beam computed tomography and radiographs in dentistry: aspects related to radiation dose. International journal of dentistry, 2012.

3. American Academy of Oral and Maxillofacial Radiology. (2013). Clinical recommendations regarding use of cone beam computed tomography in orthodontics. Position statement by the American Academy of Oral and Maxillofacial Radiology. Oral Surg Oral Med Oral Pathol Oral Radiol, 116(2), 238-257.

4. Rajpal K, Agarwal R, Chhabra R, Maumita Bhattacharya M( 2014) .Updated Classification Schemes forFibro-Osseous Lesionsof theOral \& Maxillofacial Region:A Review .Journal of Dental and Medical Sciences ,13, ( 2), 99-103

5. Barnes L, Eveson JW, Reichart P, Sidransky D. (2005) World Health Organization Classification of tumours. pathology and genetics; head a nd neck tumours; pathology and genetics; head and neck tumours. Lyon: IARC, 287-9

6. Kramer, I. R. H., Pindborg, J. J., \& Shear, M. (1992). Histological typing of odontogenic tumors. World Health Organization. International Histological Classification of Tumors.

7. Miyake, M., \& Nagahata, S. (1999). Florid cemento-osseous dysplasia: Report of a case. International Journal of Oral \& Maxillofacial Surgery, 28(1), 56-57.
8. Cannon, J. S., JS, C., EE, K., \& DC, D. (1980). Gigantiform cementoma: report of two cases (mother and son). Oral and Maxillofacial Pathology - E-Book,674.

9. Toffanin, A., Benetti, R., \& Manconi, R. (2000). Familial florid cemento-osseous dysplasia: a case report. Journal of oral and maxillofacial surgery, 58(12), 14401446.

10. Gariba-Silva, R., Sousa-Neto, M. D., Carvalho, J. J., Saquy, P. C., \& Pecora, J. D. (1999). Periapical cemental dysplasia: case report. Brazilian dental journal, 10(1), 55-57.

11. Said-al-Naief, N. A., \& Surwillo, E. (1999). Florid osseous dysplasia of the mandible: report of a case. Compendium of continuing education in dentistry (Jamesburg, NJ: 1995), 20(11), 1017-9.

12. Damm, D. D., \& Fantasia, J. E. (2001). Multifocal mixed radiolucencies. Florid cemento-osseous dysplasia. General dentistry, 49(5), 461-538.

13. Waldron CA (1993). Fibro-osseous lesions of the jaws. Journal of Oral and Maxillofacial Surgery, 51(8):828-35.

14. Singer, S. R., Mupparapu, M., \& Rinaggio, J. (2005). Florid cemento-osseous dysplasia and chronic diffuse osteomyelitis: report of a simultaneous presentation and review of the literature. The journal of the American Dental Association, 136(7), 927-931.

15. Alsufyani, N. A., \& Lam, E. W. N. (2011). Cemento-osseous dysplasia of the jaw bones: key radiographic features. Dentomaxillofacial Radiology, 40(3), 141-146.

16. Consolaro, A., Paschoal, S. R. B., Ponce, J. B., \& Miranda, D. A. O. (2018). Florid cemento-osseous dysplasia: a contraindication to orthodontic treatment in compromised areas. Dental press journal of orthodontics, 23(3), 26-34. 